\title{
Evaluación del contenido de calcio en leche y yogurt
}

\section{Evaluation of calcium content in milk and yogurt}

\author{
Alondra S. Beltrán-Aguilar ${ }^{a}$, Carolina M. Dorantes-Olvera ${ }^{b}$, Ingrid Reyes-Cano ${ }^{c}$, Samantha \\ G. Sandoval-Mejía ${ }^{d}$ y Esther Ramírez-Moreno ${ }^{e .}$
}

\begin{abstract}
:
Milk and yogurt are considered the best dietary source of calcium due to the content and bioavailability of the mineral, which is essential for muscle contraction and the function of the nervous system, therefore this nutrient must be provided in the diet. To cover the calcium recommendations, not only is food containing this mineral required, but also that calcium is bioavailable, that is, it can be absorbed in the intestine and, therefore, be used for physiological functions. The objective of this study was to evaluate and compare the calcium content in milk and yogurt to determine which product has the highest content of this mineral. The milks and yogurts evaluated presented a calcium contribution of 110-120 mg / $100 \mathrm{~mL}$, however, the yogurts presented a higher content. Therefore, these products can be considered as a good source of calcium in adults and children with contributions between 28 and $14 \%$ respectively, of the daily recommendations if $250 \mathrm{~mL}$ of product are consumed per day
\end{abstract}

Keywords:

Milk, yogurt, calcium

Resumen:

La leche y el yogurt se consideran la mejor fuente dietética de calcio por el contenido y la biodisponibilidad del mineral, el cual es imprescindible para la contracción muscular y la función del sistema nervioso, por ello este nutriente debe aportarse en la dieta. Para cubrir las recomendaciones de calcio, no solo se requiere de alimentos que contengan este mineral, sino que, además, el calcio esté biodisponible, es decir, que pueda ser absorbido en el intestino y, por lo tanto, ser utilizado para las funciones fisiológicas. El objetivo del presente estudio fue evaluar y comparar el contenido de calcio en leche y yogur para determinar qué producto tiene mayor contenido de este mineral. Las leches y los yogures evaluados presentaron un aporte de calcio de 110-120 mg/100 mL, sin embargo, los yogures presentaron un mayor contenido. Por tanto, estos productos pueden ser considerados como una buena fuente de calcio en adultos y niños con aportes entre un 28 y $14 \%$ respectivamente, de las recomendaciones diarias si se consumen 250 mL de producto al día.

\section{Palabras Clave:}

Leche, yogurt, calcio.

\footnotetext{
Autor de Correspondencia, Universidad Autónoma del Estado de Hidalgo, http://orcid.org/0000-0002-6288-9488, Email: be298098@uaeh.edu.mx

b Universidad Autónoma del Estado de Hidalgo, Instituto de Ciencias de la Salud, http://orcid.org/0000-0002-3506-8393, Email: do376084@uaeh.edu.mx

c Universidad Autónoma del Estado de Hidalgo, Instituto de Ciencias de la Salud, https://orcid.org/0000-0003-0186-8904, Email: re312950@uaeh.edu.mx

d Universidad Autónoma del Estado de Hidalgo, Instituto de Ciencias de la Salud, https://orcid.org/0000-0001-9973-719X, Email: sa287108@uaeh.edu.mx

e Universidad Autónoma del Estado de Hidalgo, Instituto de Ciencias de la Salud, https://orcid.org/0000-0002-9928-8600, Email: esther_ramirez@uaeh.edu.mx
}

Fecha de recepción: 23/10/2020, Fecha de aceptación: 04/12/2020, Fecha de publicación: 05/06/2021 


\section{Introducción}

La principal fuente de calcio en la dieta es la leche y sus derivados, aportando alrededor del $40 \%$ de las ingestas diarias recomendadas (IDR) ${ }^{1}$ y, en segundo lugar, es la carne el pollo, el pescado sobre todo lo que se consume con cartílago como la sardina, sin embargo, estos alimentos se consumen en pocas cantidades y contribuyen poco a satisfacer las IDR ${ }^{2}$. El $99 \%$ del calcio se encuentra en el esqueleto constituyendo la masa ósea de los animales, es imprescindible para la contracción muscular y la función del sistema nervioso. ${ }^{1,3}$

La Tabla 1 describe la cantidad de calcio que se establece para cada uno de los grupos etarios de acuerdo con el libro de recomendaciones de ingestión de nutrimentos para la población mexicana.

Tabla 1. Ingesta Diaria Recomendada (IDR $)^{4}$.

\begin{tabular}{ll}
\hline Edad & $\begin{array}{l}\text { Requerimiento } \\
\text { nutricional }(\mathbf{m g})\end{array}$ \\
\hline 2-8 años & 800 \\
\hline 9-18 años & 1300 \\
\hline 19-50 años & 1000 \\
\hline 51-70 años & 1200 \\
\hline $\begin{array}{l}\text { Embarazadas y } \\
\text { lactantes }\end{array}$ & 1000 \\
\hline
\end{tabular}

Para cubrir las recomendaciones de calcio, no solo se requiere de alimentos que contengan este mineral, sino que, además, el calcio esté biodisponible, es decir, que pueda ser absorbido en el intestino y, por lo tanto, ser utilizado para las funciones fisiológicas. La absorción intestinal del calcio dietético puede oscilar entre el 20 y el $75 \%$, dependiendo de la cantidad, de la forma química en la que se encuentre, de la matriz del alimento y de la presencia de otros componentes en el alimento como la lactosa y ciertos aminoácidos que facilitan su absorción. En la biodisponibilidad de este mineral influyen la caseína, lactosa, fósforo, algunos aminoácidos como lisina y arginina (transportando el calcio a través de la membrana de las células intestinales) y compuestos bioactivos, así como la edad del individuo. ${ }^{2,5}$

La biodisponibilidad de calcio puede estar aumentada o disminuida debido a que este se encuentra unido a péptidos y proteínas y es más probable que se mantenga en solución cuando el pH es desfavorable, pero también puede deberse a que se absorbe en ausencia de vitamina $\mathrm{D}$, por elementos como lactosa o lactoalbúmina ${ }^{6}$. Para establecer las IDR de este mineral se deben de tomar en cuenta factores que favorecen la disponibilidad de calcio como algunas hormonas (estrógenos y la hormona de crecimiento), aporte adecuado de vitamina $D$ y actividad física regular. ${ }^{2}$

Dentro de los factores que limitan la absorción de calcio se encuentran la deficiencia de vitamina D, la presencia de oxalatos, fitatos, fosfatos y grasas no absorbidas, una inadecuada relación calcio/fósforo en la dieta (la correcta en el adulto es de entre $2 / 1$ y $1 / 1$ ), el exceso de sodio y el consumo de café mayor a $90 \mathrm{mg} / \mathrm{día}$ (sobre todo en adolescentes) y de otras bebidas con elevado contenido de bases xánticas, que provocan hipercalciuria y aumentan la eliminación fecal de calcio. ${ }^{7}$

Objetivo: Revisar el contenido de calcio de productos lácteos: leche y yogurt, considerando la información presente en su etiquetado

\section{Metodología}

Se realizó la compilación de la información nutrimental de algunos productos lácteos (leche entera, deslactosada, yogurt natural y yogur natural griego) en supermercados convencionales dentro de la localidad de Pachuca Hidalgo. Se analizaron las etiquetas de 6 productos de yogurt marca Vitalinea, Lala y Yoplait; de leche 7 productos marca Alpura, Santa Clara y Lala. Se registró la información en el programa Microsoft Excel considerando $100 \mathrm{~g}$ o $\mathrm{mL}$ de producto para establecer una comparación entre dichos productos sobre la cantidad de calcio establecido en las etiquetas.

Las marcas fueron elegidas de acuerdo a la popularidad que tienen en el mercado, ya que son marcas que la mayoría de la población mexicana consumen. Cabe mencionar que estos productos no son aptos para todas las edades, por ejemplo, no se recomienda el yogur sin azúcar o productos light para los niños ya que se encuentran en pleno desarrollo y si lo consumen de manera cotidiana le están restando nutrientes esenciales a su alimentación.

\section{Resultados y discusión}

En la Tabla 2 se presentan las diferentes marcas de productos lácteos analizados y comparadas en relación con su contenido energético y de calcio por cada $100 \mathrm{~mL}$, así como su precio.

Los productos evaluados presentaron un aporte de calcio de $110-120 \mathrm{mg} / 100 \mathrm{~mL}$ y solo la leche Lala $\mathrm{sin}$ lactosa más calcio presenta un contenido mayor (160 $\mathrm{mg} / 100 \mathrm{~mL}$ ). En el caso de los yogures presentaron un mayor contenido alrededor de 142 y $144 \mathrm{mg} / 100 \mathrm{~mL}$ con excepción de uno de los productos que presentó $52 \mathrm{mg}$. Por lo tanto, de acuerdo a Farré (2015) estos productos lácteos pueden ser considerados como una buena fuente de calcio con aportes de 120 a $144 \mathrm{mg} / 100 \mathrm{~mL}$ si se 
consumen al menos $250 \mathrm{~mL}$ de producto al día. De acuerdo a este contenido de calcio el consumo de estos productos lácteos puede contribuir entre 18 y $28 \%$ de las IDR para niños entre $1-8$ años y 11 y $14 \%$ en adolescentes, adultos y embarazadas. Cabe destacar que a pesar de ser una de las mejores fuentes de calcio, no se recomienda su consumo en niños menores de 1 año ya que no se puede comparar con todos los nutrientes que aporta la leche materna. ${ }^{4}$ Después del primer año de vida, la ingesta de 2-3 raciones diarias de leche (400 a $550 \mathrm{~mL}$ ) de vaca, contribuye en gran parte a cubrir las necesidades de calcio en el niño. ${ }^{4}$

Tabla 2 Comparación de datos obtenidos de diferentes marcas de leche y yogurt.

\begin{tabular}{|c|c|c|c|c|c|c|c|c|c|c|}
\hline \multicolumn{11}{|c|}{ Leche } \\
\hline Marca & $\mathrm{ml}$ & & $\begin{array}{l}\text { Energía } \\
\text { (kcal) }\end{array}$ & $\begin{array}{l}\text { Proteina } \\
\text { s } \\
(g r)\end{array}$ & $\begin{array}{c}\text { Lipidos } \\
\text { (gr) }\end{array}$ & $\begin{array}{c}\text { Grasas } \\
\text { saturada } \\
\text { s } \\
(\mathrm{gr})\end{array}$ & $\begin{array}{ll}\mathrm{HCO} & A \\
(\mathrm{gr}) & \end{array}$ & $\begin{array}{c}\text { Azucares } \\
\text { (gr) }\end{array}$ & $\begin{array}{l}\text { Calcio } \\
(\mathrm{mg})\end{array}$ & Precio \\
\hline $\begin{array}{c}\text { Santa Clara } \\
\text { entera }\end{array}$ & 100 & & 61.4 & 2.66 & 3 & 1.84 & 5.6 & 4.8 & 119.6 & 22 \\
\hline $\begin{array}{l}\text { Alpura } \\
\text { entera }\end{array}$ & 100 & & 56.6 & 3.1 & 2.7 & 1.8 & 4.7 & 4.7 & 110 & 21 \\
\hline $\begin{array}{c}\text { Lala sin } \\
\text { lactosa, má } \\
\text { calcio }\end{array}$ & as 100 & & 43.7 & 5.4 & 1 & 0.6 & 3.3 & 3.3 & 160 & 27 \\
\hline Lala entera & (a) 100 & & 41.5 & 3.1 & 3.3 & 2.0 & 4.8 & 4.8 & 116 & 21 \\
\hline $\begin{array}{l}\text { Santa clara } \\
\text { deslactorad }\end{array}$ & das 100 & & 50.5 & 3 & 1.8 & 1 & 5.6 & 4.4 & 119.6 & 24 \\
\hline $\begin{array}{c}\text { Lala } \\
\text { deslactosad }\end{array}$ & do 100 & & 48 & 3.1 & 0.5 & 0.3 & 4.8 & 4.8 & 122.3 & 21.5 \\
\hline \multicolumn{11}{|c|}{$\begin{array}{c}\text { Alpura } \\
\text { deslactosada }\end{array}$} \\
\hline \multicolumn{11}{|c|}{ Yogurt } \\
\hline Marca & Sabor & Kcal & II Gramos & $\begin{array}{l}\text { Proteinas } \\
(\mathrm{gr})\end{array}$ & $\begin{array}{l}\text { Lipidos } \\
\text { (gr) }\end{array}$ & $\begin{array}{c}\text { Grasas } \\
\text { saturadas } \\
\text { (gr) }\end{array}$ & $\begin{array}{r}\mathrm{HCO} \\
5 \quad(\mathrm{gr}) \\
\end{array}$ & $\begin{array}{c}\text { Azucares } \\
\text { (grt) }\end{array}$ & $\begin{array}{l}\text { Calcio } \\
(\mathrm{mg})\end{array}$ & Precio \\
\hline Vitalinea & Natural & 58 & 100 & 3.8 & 1.4 & 0.9 & 7.1 & 6 & 142.7 & 4.8 \\
\hline $\begin{array}{c}\text { Vitalinea } \\
\text { griego }\end{array}$ & Natural & 61.6 & 100 & 7.6 & 1.5 & 0.9 & 4.3 & 3.9 & 139.7 & 12 \\
\hline Lala & Natural & 140 & 100 & 3.5 & 2 & 1.2 & 13.0 & 12.1 & 52 & 5.2 \\
\hline $\begin{array}{l}\text { Lala } \\
\text { griego }\end{array}$ & Natural & 81.6 & 100 & 5.2 & 7 & 4.3 & 15.4 & 14.9 & 72.8 & 11.4 \\
\hline Yoplait & Natural & 94.4 & 100 & 3.2 & 2.2 & 1.2 & 15.2 & 12 & 128 & 5.8 \\
\hline
\end{tabular}

Además del calcio, la leche tiene un aporte calórico de 41$61 \mathrm{kcal}$, entre 2.6 a $5.4 \mathrm{~g}$ de proteína, lípidos 2.7 a $3.3 \mathrm{~g}$ y $2.04 \mathrm{~g}$ de grasas saturadas en $100 \mathrm{~mL}$, con excepción de las leches descremadas que por norma debe tener entre 0.6 y $2.8 \mathrm{~g} / 100 \mathrm{~mL}$ o semidescremadas $(0.5 \mathrm{~g}) .^{8}$ También aporta carbohidratos, caracterizados principalmente como lactosa (3-5 g). En el caso del yogurt su aporte calórico y proteico es mayor que la leche por el contenido de azúcares y la concentración de proteínas que se lleva a cabo para la obtención del yogurt griego. De acuerdo con el CODEX STAN 243-2003, todos los yogures se encuentran dentro del valor establecido, ${ }^{9}$ a excepción de los yogures naturales (Lala griego, Vitalinea y Yoplait natural) que presentan un alto contenido de azúcares. De acuerdo con la NOM-051-SCFI/SSA1-2010 y su última actualización, se menciona que tiene por objeto establecer la información comercial y sanitaria que debe contener el etiquetado del producto preenvasado destinado al consumidor final, de fabricación nacional o extranjera, comercializado en territorio nacional, así como determinar las características de dicha información y establecer un sistema de etiquetado frontal, el cual debe advertir de forma clara y veraz sobre el contenido de nutrientes críticos e ingredientes que representan riesgos para su salud en un consumo excesivo. Por lo que los productos de analizados en este artículo que tienen un alto contenido de azúcares (mayor o igual al 10\% del total de energía proveniente de azúcares libres, es decir monosacáridos y disacáridos disponibles añadidos a los alimentos y a las bebidas no alcohólicas, más los azúcares que están presentes naturalmente en miel, jarabes y jugos de frutas $\mathrm{u}$ hortalizas), ${ }^{10}$ deben tener un sello rectangular de advertencia y, por lo tanto, su consumo debe ser moderado o buscar presentaciones con bajo contenido de azúcar.

\section{Conclusiones}

Los productos lácteos (leche y yogur) en general tienen un buen aporte proteínas y calcio, sin embargo, hay que considerar los productos que tengan el menor contenido de azúcares libres ya que su consumo contribuye a la densidad calórica total de la dieta, y el consumo excesivo pone en peligro la calidad nutricional de la dieta al aportar muchas calorías sin nutrientes específicos, y esto puede producir un aumento de peso y un aumento del riesgo de obesidad y diversas enfermedades.

Las leches y los yogures comerciales evaluados en este trabajo cubrieron al menos $110 \mathrm{mg}$ de calcio por $100 \mathrm{~g}$ de producto, por lo que el consumo al menos de 250 $\mathrm{mL}$ en escolares y adultos cubren entre un 28 y $14 \%$ respectivamente.

El calcio presente en estos productos tiene una alta biodisponibilidad en el organismo y esto permite que tengan efectos positivos en la salud por lo que la recomendación es de al menos $250 \mathrm{~mL}$ diarios.

\section{Referencias}

[1] Martínez E. El calcio, esencial para la salud. Nutr Hosp. (2016); 33 (4):26-31.

[2] Rodríguez-Huertas J, Rodríguez Lara A, González- Acevedo O, Dolores Mesa M. Leche y productos lácteos como vehículos de calcio y vitamina D: papel de leches enriquecidas. Nutr. Hosp. (2019); 36 (4). Disponible en : 
Publicación semestral, Educación y Salud Boletín Científico Instituto de Ciencias de la Salud Universidad Autónoma del Estado de

Hidalgo, Vol. 9, No. 18 (2021) 114-117

http://scielo.isciii.es/scielo.php?script=sci_arttext\&pid=S0212-

16112019000400030

[3] Theobald HE. Dietary calcium and health. Nutr Bullet. (2005); 30 (3):237-277.

[4]Bourges H, Casanueva E, Rosado JL. Recomendaciones de Ingestión de Nutrimentos para la Población Mexicana. México: Panamericana; 2008

[5] Poveda Elpidia E. Suero lácteo, generalidades y potencial uso como fuente de calcio de alta biodisponibilidad. Rev. Chil. Nutr. (2013); 40 (4). Disponible en: https://scielo.conicyt.cl/scielo.php?pid=S071775182013000400011\&script=sci_arttext\&tlng=e

[6] Aznar L. A. Evidencia científica sobre el papel del yogur y otras leches fermentadas en la alimentación saludable de la población española. Nutr. Hosp (2013); 28 (6). Disponible en: http://scielo.isciii.es/scielo.php?pid=S0212$16112013000600038 \&$ script=sci_arttext\&tlng=pt

[7] Farré R. La leche y los productos lácteos: fuentes dietéticas de calcio. Nutr Hosp. (2015); 31(2). Disponible en: http://www.aulamedica.es/nh/pdf/8676.pdf

[8] NOM-155-SCFI-2012. Leche-denominaciones, especificaciones fisicoquímicas, información comercial y métodos de prueba. Disponible en:

http://www.dof.gob.mx/normasOficiales/4692/seeco/seeco.htm

[9] Codex Stan 243-2003 NORMA DEL CODEX PARA LECHES FERMENTADAS. Dirección: http://www.fao.org/3/a-i2085s.pdf

[10] NOM-051-SCFI/SSAI-2010, Especificaciones generales de etiquetado para alimentos y bebidas no alcohólicas preenvasadosinformación comercial y sanitaria. Disponible en: https://www.dof.gob.mx/2020/SEECO/NOM_051.pdf 